Pacific

Journal of

Mathematics

VIRTUAL HOMOLOGY OF SURGERED TORUS BUNDLES Joseph D. MAsters 


\title{
VIRTUAL HOMOLOGY OF SURGERED TORUS BUNDLES
}

\author{
Joseph D. MASTERS
}

Let $M$ be a once-punctured torus bundle over $S^{1}$ with monodromy $h$. We show that, under certain hypotheses on $h$, "most" Dehn-fillings of $M$ (in some cases all but finitely many) are virtually $\mathbb{Z}$-representable. We apply our results to show that even surgeries on the figure eight knot are virtually $\mathbb{Z}$-representable.

\section{Introduction.}

Embedded incompressible surfaces are fundamental in the study of 3-manifolds. Accordingly, the following conjecture of Waldhausen and Thurston has attracted much attention:

Conjecture 1.1. Let $M$ be a closed, irreducible 3-manifold with infinite $\pi_{1}$. Then $M$ has a finite cover which is Haken.

The focus of this paper is the following, stronger, conjecture:

Conjecture 1.2. Let $M$ be as above. Then $M$ has a finite cover $\tilde{M}$ with $H_{1}(\tilde{M}, \mathbb{Z})$ infinite.

If $M$ is a compact 3 -manifold, we say that $M$ is $\mathbb{Z}$-representable if $H_{1}(M, \mathbb{Z})$ is infinite. If $M$ satisfies the conclusion of Conjecture 1.2 , we say that $M$ is virtually $\mathbb{Z}$-representable.

We shall give what appear to be the first examples of 3-manifolds with torus boundary for which all but finitely many fillings are virtually $\mathbb{Z}$ representable, but not $\mathbb{Z}$-representable (in fact non-Haken). Boyer and Zhang have independently given examples of knot complements for which all but finitely many fillings are virtually Haken, but non-Haken [BZ].

Before we can state our results, we must establish some notation. Let $F$ be a once-punctured torus with $\pi_{1}(F)=\langle[x],[y]\rangle$, and basepoint $x_{0} \in \partial F$ (see Fig. 1).

Any orientation-preserving homeomorphism $h: F \rightarrow F$ is isotopic to one of the form $h=D_{x}^{r_{1}} D_{y}^{s_{1}} \cdots D_{x}^{r_{k}} D_{y}^{s_{k}}$. Here $D_{x}$ and $D_{y}$ are Dehn twists along simple closed curves homologous to $x$ and $y$, respectively. The twists $D_{x}$ 


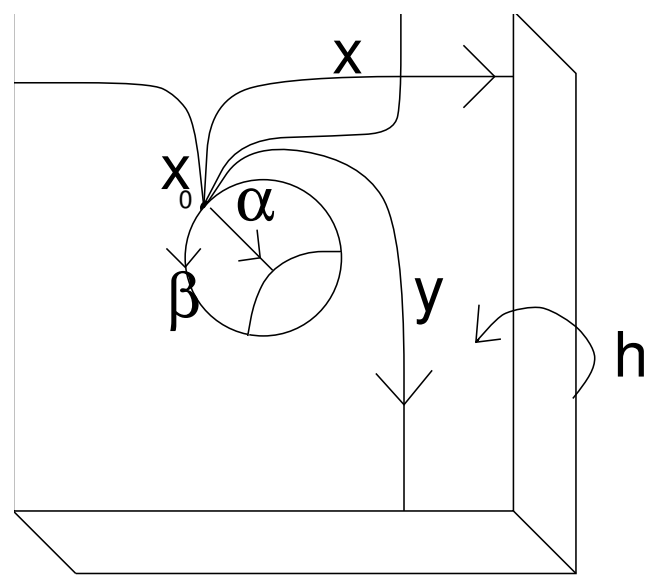

Figure 1. Notation for the once-punctured torus bundle $M$.

and $D_{y}$ induce the following actions on $\pi_{1}(F)$ :

$$
\begin{aligned}
D_{x \sharp}(x) & =x \\
D_{x \sharp}(y) & =y x \\
D_{y \sharp}(x) & =y x \\
D_{y \sharp}(y) & =y .
\end{aligned}
$$

We may assume $h$ fixes $\partial F$. Let $M_{h}=(F \times I) / h$ be the once-punctured torus bundle with monodromy $h$. We specify a framing for $H_{1}\left(\partial M_{h}, \mathbb{Z}\right)$ by setting the longitude $\beta=\partial F$ oriented counter-clockwise, and the meridian $\alpha=\left(x_{0} \times I\right) / h$, where $x_{0}$ is some point on $\partial F$, and $\alpha$ is oriented as in Fig. 1 . Then, for coprime integers $(\mu, \lambda), M_{h}(\mu, \lambda)$ denotes the manifold obtained by gluing a solid torus to $M_{h}$ in such a way that the curve $\alpha^{\mu} \beta^{\lambda}$ becomes homotopically trivial.

We shall prove:

Theorem 1.3. Let $M_{h}$ be a once-punctured torus bundle over $S^{1}$, with monodromy $h=D_{x}^{r_{1}} D_{y}^{s_{1}} \cdots D_{x}^{r_{k}} D_{y}^{s_{k}}$, and let $n=$ g.c.d $\left\{s_{1}, \ldots, s_{k}\right\}, R=$ $r_{1}+\cdots+r_{k}$.

(i) If $n$ is divisible by some $m$ such that $m \geq 6$ and $m$ is even or $m=7$, and if $|\lambda|>1$, then all but finitely many Dehn-fillings $M_{h}(\mu, \lambda)$ are virtually $\mathbb{Z}$-representable.

(ii) If $n$ is divisible by some $m$ such that $m \geq 5, m$ is odd, and $m \neq 7$, and if $1 /|R \mu-\lambda|+1 /|R \mu-2 \lambda|+1 /|\lambda|<1$, then $M_{h}(\mu, \lambda)$ is virtually $\mathbb{Z}$-representable.

(iii) If $n$ is divisible by 4 , and if $2 /|R \mu-2 \lambda|+1 /|\lambda|<1$, then $M_{h}(\mu, \lambda)$ is virtually $\mathbb{Z}$-representable. 
Remarks. 1. Analogous results hold if we replace $n$ by $g c d\left\{r_{1}, \ldots, r_{k}\right\}$ and $R$ by $s_{1}+\cdots+s_{k}$.

2. It was shown in [B1] that if $m \geq 2, n \geq 2$ and $m n \geq 8$ but $m n \neq 9$, then all non-integral surgeries are virtually $\mathbb{Z}$-representable. In [B2] it was shown that if $4 \mid n$, then for each $\mu, M_{h}(\mu, \lambda)$ is virtually $\mathbb{Z}$-representable for all but finitely many $\lambda$ coprime to $\mu$.

3. From $[\mathbf{C J R}]$ and $[\mathbf{F H}]$, all but finitely many surgeries on a oncepunctured torus bundle over $S^{1}$ yield non-Haken manifolds.

Theorem 1.3 may be used to show that, for certain choices of $f$, all but finitely many surgeries on $M_{f}$ are virtually $\mathbb{Z}$-representable. For example:

Theorem 1.4. Let $f=\left(D_{x} D_{y}\right)^{18}$. Then every surgery on $M_{f}$ is virtually $\mathbb{Z}$-representable.

The proof of Theorem 1.4 appears in Section 3.

In order to state the next theorem, we require some notation. Let $-1=$ $\left(D_{x} D_{y}^{-1} D_{x}\right)^{2}$, the central involution on the punctured torus. If $h$ is a homeomorphism of the punctured torus, $-h$ stands for $(-1) h$.

Theorem 1.5. Let $N=M_{-D_{x} D_{y}}$ (also known as "the figure eight knot's sister"). Then if $1 /|\mu-\lambda|+1 /|\mu-2 \lambda|+1 /|\lambda|<1, N(\mu, \lambda)$ is virtually $\mathbb{Z}$-representable.

Theorem 1.6. Let $K$ denote the figure-eight knot and let $M$ denote $S^{3}-K$. Then, with respect to the canonical framing of knots in $S^{3}$, any surgery of the form $M(2 \mu, \lambda)$ is virtually $\mathbb{Z}$-representable.

Other results on virtually $\mathbb{Z}$-representable figure-eight knot surgeries may be found in $[\mathbf{M}],[\mathbf{K L}],[\mathbf{H}],[\mathbf{N}]$ and $[\mathbf{B 3}]$. In particular, it was shown in $[\mathbf{K L}]$ and [B3] that surgeries of the form $M(4 \mu, \lambda)$ are virtually $\mathbb{Z}$-representable. It was also shown in [B3] that surgeries of the form $M(2 \mu, \lambda)$ are virtually $\mathbb{Z}$-representable if $\lambda= \pm 7 \mu(\bmod 15)$. Finally, it was shown in [Bart] that every non-trivial surgery of $M$ contains an immersed incompressible surface.

Our techniques are extensions of Baker's. The main new ingredient is the use of group theory to encode the combinatorics of cutting and pasting.

I would like to thank Professor Alan Reid for his help and patience.

\section{Construction of covers.}

We begin by recalling Baker's construction of covering spaces of $M_{h}(\mu, \lambda)$ (see $[\mathbf{B 1}],[\mathbf{B 2}])$. Let $n$ be as in the statement of Theorem 1.3, and let $\hat{F}$ be the $k n$-fold cover of $F$ associated to the kernel of the map $\phi: \pi_{1}(F) \rightarrow$ $\mathbb{Z}_{k} \times \mathbb{Z}_{n}$, with $\phi([x])=(1,0)$ and $\phi([y])=(0,1)$ (see Fig. 2).

Now create a new cover, $\tilde{F}$, of $F$ by making vertical cuts in each row of $\hat{F}$, and gluing the left side of each cut to the right side of another cut in the 


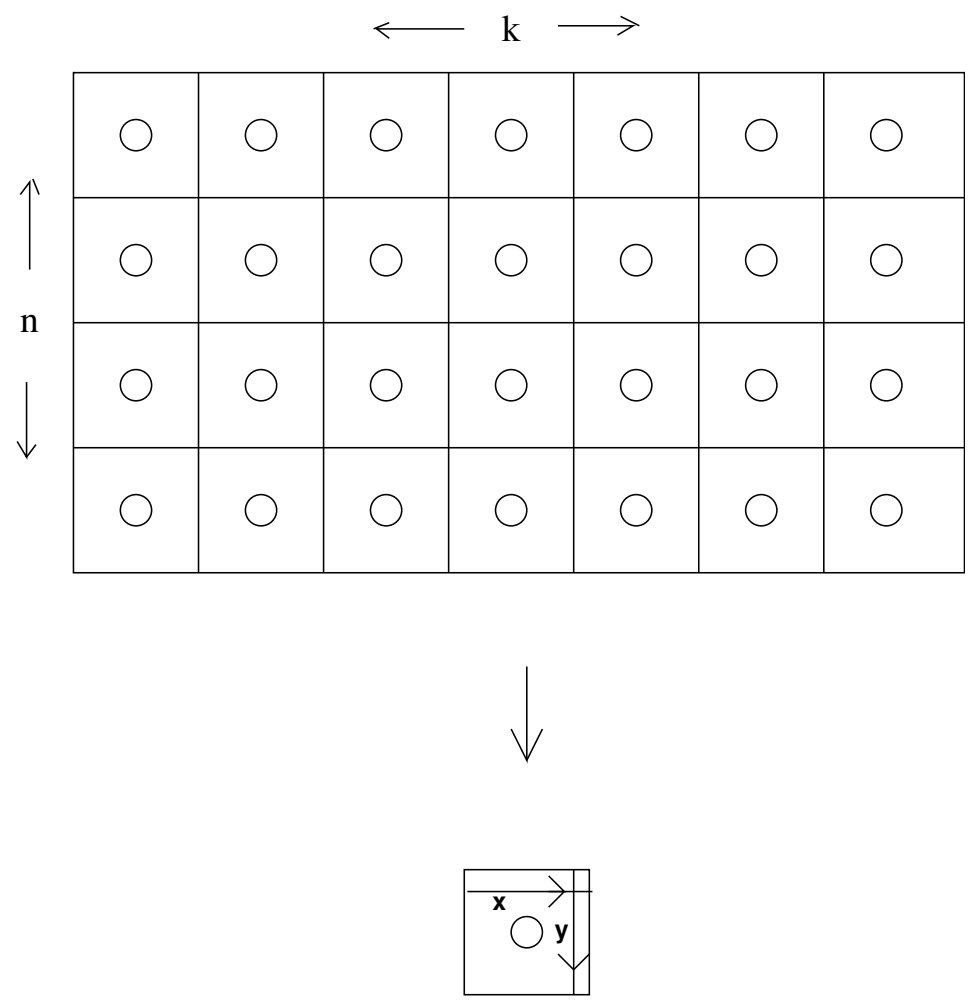

Figure 2. The cover $\hat{F}$ of $F$.

same row. An example is pictured in Figure 3, where the numbers in each row indicate how the edges are glued.

If $h$ lifts to a map $\tilde{h}: \tilde{F} \rightarrow \tilde{F}$, then the mapping cylinder $\tilde{M}_{h}=\tilde{F} / \tilde{h}$ is a cover of $M_{h}$. Furthermore, if the loop $\alpha^{\mu} \beta^{\lambda}$ lifts to loops in $\tilde{M}_{h}$, then the cover extends to a cover $\tilde{M}_{h}(\mu, \lambda)$ of $M_{h}(\mu, \lambda)$.

If the cover $\tilde{M}_{h}$ exists, then we may compute its first Betti number with the formula $b_{1}\left(\tilde{M}_{h}\right)=\operatorname{rank}\left(f i x\left(\tilde{h}_{*}\right)\right)$, where $\tilde{h}_{*}$ is the map on $H_{1}(\tilde{M}, \mathbb{Z})$ induced by $\tilde{h}$, and $\operatorname{fix}\left(\tilde{h}_{*}\right)$ is the subgroup of $H_{1}(\tilde{M}, \mathbb{Z})$ fixed by $\tilde{h}_{*}$ (see $[\mathbf{H}]$ for a proof). We shall use this formula to prove that, in some cases, $b_{1}(\tilde{M})$ is greater than the number of boundary components of $\tilde{M}$, which ensures that $b_{1}(\tilde{M}(\mu, \lambda))>0$.

We now introduce some notation to describe the cuts of $\tilde{F}$ (see Fig. 3 ). $\tilde{F}$ is naturally divided into rows, which we label $1, \ldots, n$. The cuts divide each row into pieces, each of which is a square minus two half-disks; we number them $1, \ldots, k$. If we slide a point in the top half of the $i^{t h}$ row through the cut to its right, we induce a permutation on $\{1, \ldots, k\}$, which we denote 
Piece 3, Row 1

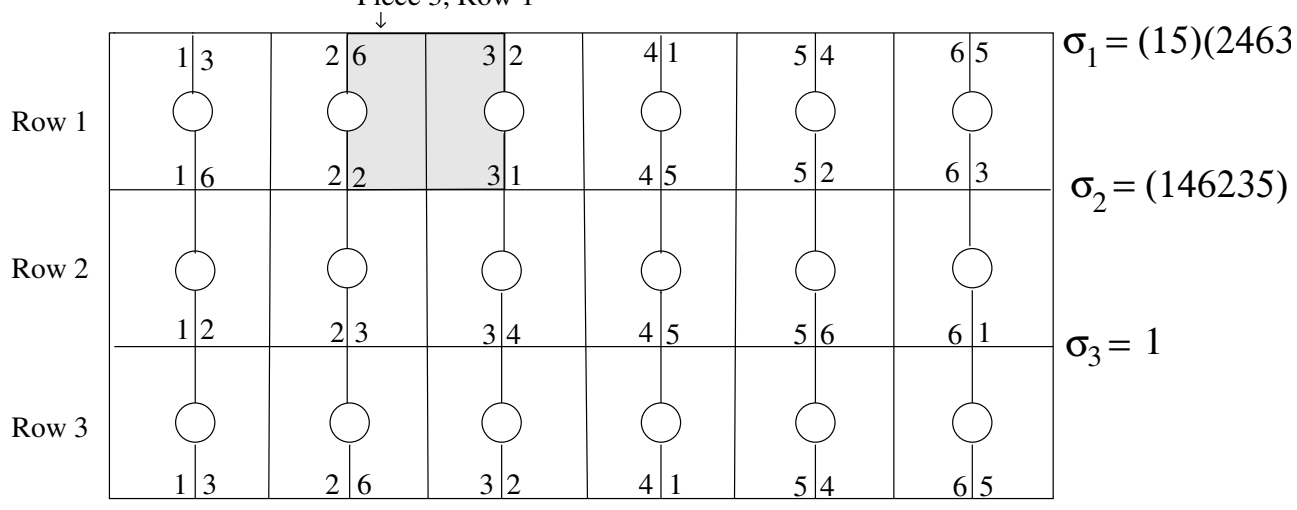

Figure 3. The permutations encode the combinatorics of the gluing.

$\sigma_{i}$. Thus the cuts on $\tilde{F}$ may be encoded by elements $\sigma_{1}, \ldots, \sigma_{n} \in S_{k}$, the permutation group on $k$ letters.

Next, we find algebraic conditions on the $\sigma_{i}$ 's which will guarantee that the cover of $F$ extends to a cover of $M(\mu, \lambda)$. We first must pick $k, n$, and $\left\{\sigma_{1}, \ldots, \sigma_{n}\right\}$ so that $h$ lifts to $\tilde{F}$.

\section{Lemma 2.1. If}

I. $\left[\sigma_{i}, \sigma_{1} \sigma_{2} \cdots \sigma_{i-1}\right]=1$ for all $i$ and

II. $\sigma_{1} \sigma_{2} \cdots \sigma_{n}=1$

then $h$ lifts to $\tilde{F}$.

Proof. Note that $D_{y}^{n}$ lifts to Dehn twists on $\tilde{F}$. Therefore, we need only ensure that $D_{x}$ lifts. We shall attempt to lift $D_{x}$ to a sequence of "fractional Dehn twists" along the rows of $\tilde{F}$. Let $\tilde{x}_{i}$ denote the disjoint union of the lifts of $x$ to the $i^{t h}$ row of $\tilde{F}$. We first attempt to lift $D_{x}$ to row 1 , twisting $1 / k^{t h}$ of the way along $\tilde{x}_{1}$. Considering the action on the bottom half of row 1 , we find that the cuts are now matched up according to the permutation $\sigma_{1}^{-1} \sigma_{2} \sigma_{1}$. Thus, for $D_{x}$ to lift to row 1 we assume $\sigma_{1}$ and $\sigma_{2}$ commute. We now twist along $\tilde{x}_{2}$. The top halves of the squares in row 2 are moved according to the permutation $\sigma_{1} \sigma_{2}$, and the lift will extend to all of row 2 if and only if $\sigma_{3}$ commutes with $\sigma_{1} \sigma_{2}$. We continue in this manner, obtaining the conditions in I. After we twist through $\tilde{x}_{n}$, we need to be back where we started in row 1 , so we require the additional condition $\sigma_{1} \sigma_{2} \cdots \sigma_{n}=1$.

Note that the loop $\alpha^{\mu}$ lifts homeomorphicly to loops in $\tilde{M}_{h}$ if $\tilde{h}^{\mu}=i d$, and that the loop $\beta^{\lambda}$ lifts to loops in $\tilde{M}_{h}$ if $\left(\sigma_{i+1} \sigma_{i}^{-1}\right)^{\lambda}=i d$ for all $i=1, \ldots, n$. Then, by considering the action of $\tilde{h}$ on $\tilde{M}_{h}$, the following condition for a loop in $\partial M_{h}$ to lift to $\tilde{M}_{h}$ is easily verified: 
Lemma 2.2. The loop $\alpha^{\mu} \beta^{\lambda} \subset \partial M_{h}$ lifts homeomorphicly to loops in $\tilde{M}_{h}$ if and only if

III. $\left(\sigma_{1} \cdots \sigma_{i}\right)^{R \mu}\left(\sigma_{i+1} \sigma_{i}^{-1}\right)^{\lambda}=1$, for $i=1, \ldots, n$.

Therefore we may construct covers of $M_{h}(\mu, \lambda)$ simply by finding permutations satisfying conditions I-III.

Proof of Theorem 1.3.

Case 1. $m=4$.

Construction of the cover of $M_{h}(\mu, \lambda)$.

To construct a cover of $M_{h}(\mu, \lambda)$, we must first construct a cover of $F$. It was shown in the discussion prior to Lemma 2.1 that there is a unique such cover associated to any four permutations $\sigma_{1}, \sigma_{2}, \sigma_{3}$ and $\sigma_{4}$ in any permutation group $S_{k}$.

To ensure that the cover of $F$ extends to a cover of $M_{h}$, we shall set $\sigma_{2}=\sigma_{1}^{-1}$ and $\sigma_{4}=\sigma_{3}^{-1}$ (see Fig. 4a). Then conditions I and II of Lemma 2.1 are satisfied automatically, so that any choice of $\sigma_{1}$ and $\sigma_{3}$ will determine a cover of $M_{h}$.

To ensure that the cover extends to $M_{h}(\mu, \lambda)$, we must arrange for the surgery curve $\alpha^{\mu} \beta^{\lambda}$ to lift to $\tilde{M}_{h}$. By Lemma 2.2, $\alpha^{\mu} \beta^{\lambda}$ will lift provided that $\sigma_{1}, \ldots, \sigma_{4}$ satisfy condition III, which reduces to:

$$
\begin{gathered}
\sigma_{1}^{R \mu-2 \lambda}=1 \\
\left(\sigma_{3} \sigma_{1}\right)^{\lambda}=1 \\
\sigma_{3}^{R \mu-2 \lambda}=1 \\
\left(\sigma_{1} \sigma_{3}\right)^{\lambda}=1 .
\end{gathered}
$$

Any pair of permutations $\sigma_{1}$ and $\sigma_{3}$ satisfying Equations (1)-(4) determines a unique cover of $M_{h}(\mu, \lambda)$. We now turn our attention to the construction of such permutations.

Consider the abstract group $G$ generated by the symbols $\bar{\sigma}_{1}$ and $\bar{\sigma}_{3}$, satisfying relations (1)-(4). $G$ is a $(|R \mu-2 \lambda|,|R \mu-2 \lambda|,|\lambda|)$-triangle group. It is well-known that if $1 /|R \mu-2 \lambda|+1 /|R \mu-2 \lambda|+1 /|\lambda|<1$, then $G$ is residually finite, and hence surjects a finite group $H$ such that the images of $\bar{\sigma}_{1}, \bar{\sigma}_{3}$, and $\overline{\sigma_{3}} \overline{\sigma_{1}}$ have order $|R \mu-2 \lambda|$. By taking the permutation representation of $H$, we then obtain permutations $\sigma_{1}$ and $\sigma_{3}$ satisfying conditions (1)-(4). Note that the permutations act on $|H|$ letters, so $\tilde{M}$ is a $4|H|$-fold cover of $M_{h}$.

Associated with the permutations $\sigma_{1}$ and $\sigma_{3}$ we have covers $\tilde{M}_{h}$ and $\tilde{F}$ of $M_{h}$ and $F$, and a cover $\tilde{M}_{h}(\mu, \lambda)$ of $M_{h}(\mu, \lambda)$;

Claim. $b_{1}\left(\tilde{M}_{h}(\mu, \lambda)\right)>0$. 


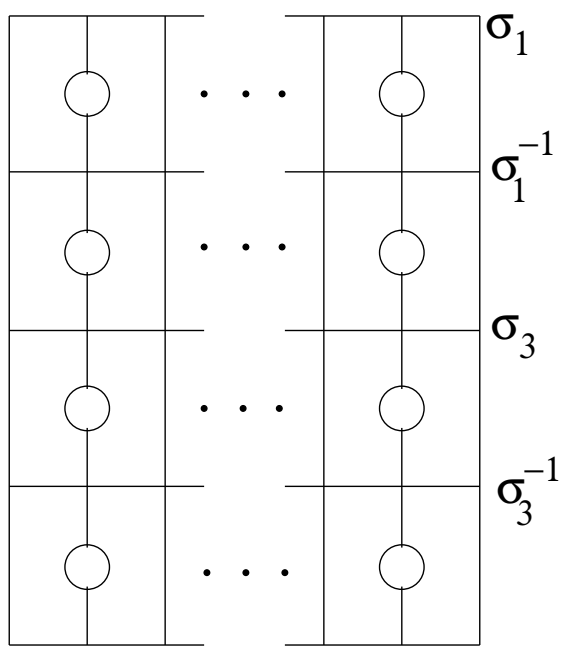

a

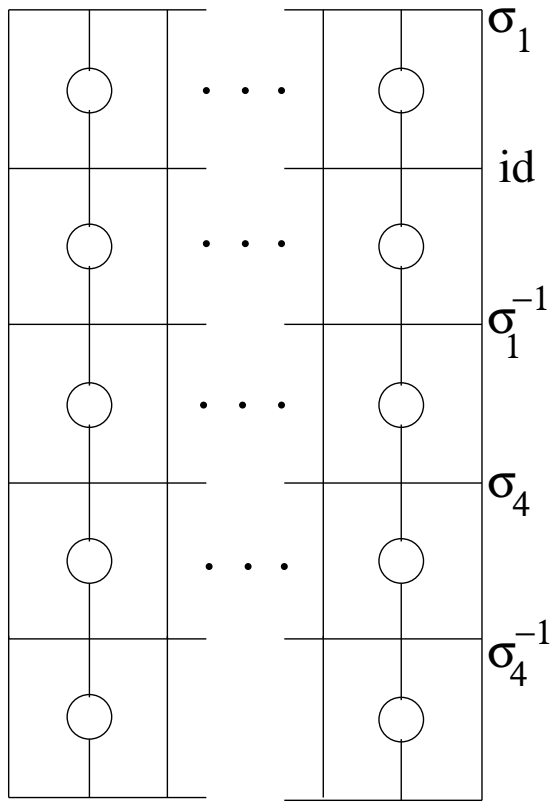

b

Figure 4
a. The cover when $n=4$.
b. The cover when $n=5$.

Proof of claim. It suffices to show that $\tilde{h}_{*}$ has a non-peripheral class $[\delta] \in$ $H_{1}(\tilde{F})$ with $\tilde{h}_{*}([\delta])=[\delta]$. To construct this element, we shall first find a non-peripheral class $\left[\delta_{2}\right]$ in row 2 , as follows.

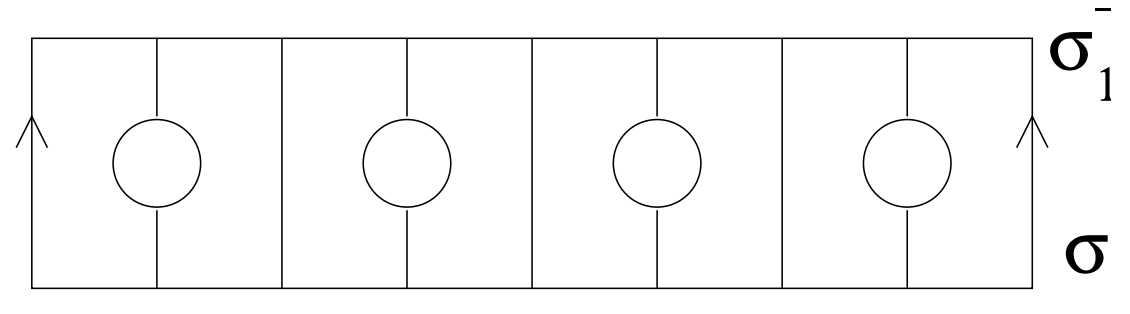

Figure 5. The surface $\tilde{F}_{2}($ with $|H|=4)$.

Consider the sub-surface $\tilde{F}_{2}$ obtained by deleting rows 1,3 and 4 from $\tilde{F}$ (see Fig. 5). The punctures of $\tilde{F}_{2}$ are in 1-1 correspondence with the cycles 
of $\sigma_{1}, \sigma_{3}$ and $\sigma_{3} \sigma_{1}$. Any permutation $\tau$ coming from the permutation representation of $H$ decomposes as a product of $|H| / \operatorname{order}(\tau)$ disjoint $\operatorname{order}(\tau)$ cycles. Therefore $\tilde{F}_{2}$ has $|H|\left(1 / \operatorname{order}\left(\sigma_{1}\right)+1 / \operatorname{order}\left(\sigma_{3}\right)+1 / \operatorname{order}\left(\sigma_{3} \sigma_{1}\right)\right)<$ $|H|$ punctures. Since $\chi\left(\tilde{F}_{2}\right)=-|H|$, we deduce that $\tilde{F}_{2}$ contains a nonperipheral class $\left[\delta_{2}\right]$. The class $\delta_{2}$ also represents a non-peripheral class in $\tilde{F}$, since it has non-zero intersection number with a class of $\tilde{F}$ in row 2 .

We may find a corresponding non-peripheral loop $\delta_{4}$ in row 4 , such that $I\left(\left[\delta_{2}+\delta_{4}\right],\left[\tilde{y}_{i}\right]\right)=0$ for all $i$ (see Fig. 6 for the notation and the idea of the proof). Let $[\delta]=\left[\delta_{2}+\delta_{4}\right]$. Then, since $[\delta]$ has non-zero intersection number with classes in row 2 and row 4 , it is a non-peripheral class. We have $I\left[\delta, \tilde{y}_{i}\right]=0$ for all $i$ (where $I(.,$.$) denotes oriented intersection number);$ therefore $[\delta]$ is fixed by $\tilde{D}_{y_{*}}^{4}$, and since $\tilde{D}_{x}$ fixes rows 2 and 4 , it is fixed by $\tilde{D}_{x *}$. Therefore it is fixed by $\tilde{h}_{*}$, concluding the proof of the claim, and of Case 1.

Case 2. $m \geq 5$ and $m$ is odd.

Case 2a. $m=5$.

The construction proceeds analogously to the case $m=4$. We require permutations $\sigma_{1}, \ldots, \sigma_{5}$ satisfying conditions I-III. Again, to simplify matters, we shall impose some extra conditions: $\sigma_{2}=i d, \sigma_{3}=\sigma_{1}^{-1}$, and $\sigma_{5}=\sigma_{4}^{-1}$ (see Fig. 4). Then conditions I-III reduce to:

$$
\begin{aligned}
\sigma_{1}^{R \mu-\lambda} & =1 \\
\left(\sigma_{1} \sigma_{4}\right)^{\lambda}=\left(\sigma_{4} \sigma_{1}\right)^{\lambda} & =1 \\
\sigma_{4}^{R \mu-2 \lambda} & =1 .
\end{aligned}
$$

Again, these relations determine a triangle group, which, under the hypotheses on $\mu$ and $\lambda$, is hyperbolic. The rest of the proof is identical to Case 1, except that now the fixed class is in rows 3 and 5 .

Case 2 b. $m \geq 9$ and $m$ is odd.

Consider the cover obtained by setting $\sigma_{2}=\sigma_{1}^{-1}, \sigma_{4}=i d, \sigma_{5}=\sigma_{3}^{-1}$, $\sigma_{6}=\sigma_{1}, \sigma_{7}=\sigma_{1}^{-1}$, and for $i=4, \ldots, k, \sigma_{2 i+1}=\sigma_{2 i}^{-1}$ (see Fig. 7a).

The corresponding relations are:

$$
\begin{gathered}
\sigma_{1}^{R \mu-2 \lambda}=1 \\
\sigma_{3}^{R \mu-\lambda}=1 \\
\left(\sigma_{3} \sigma_{1}\right)^{\lambda}=\left(\sigma_{1} \sigma_{3}\right)^{\lambda}=1 \\
\left(\sigma_{8} \sigma_{1}\right)^{\lambda}=1 \\
\sigma_{2 i}^{R \mu-2 \lambda}=1 \text { for } i=4, \ldots, k \\
\left(\sigma_{2 i+2} \sigma_{2 i}\right)^{\lambda}=1 \text { for } i=4, \ldots, k-1 \\
\left(\sigma_{1} \sigma_{2 k}\right)^{\lambda}=1 .
\end{gathered}
$$



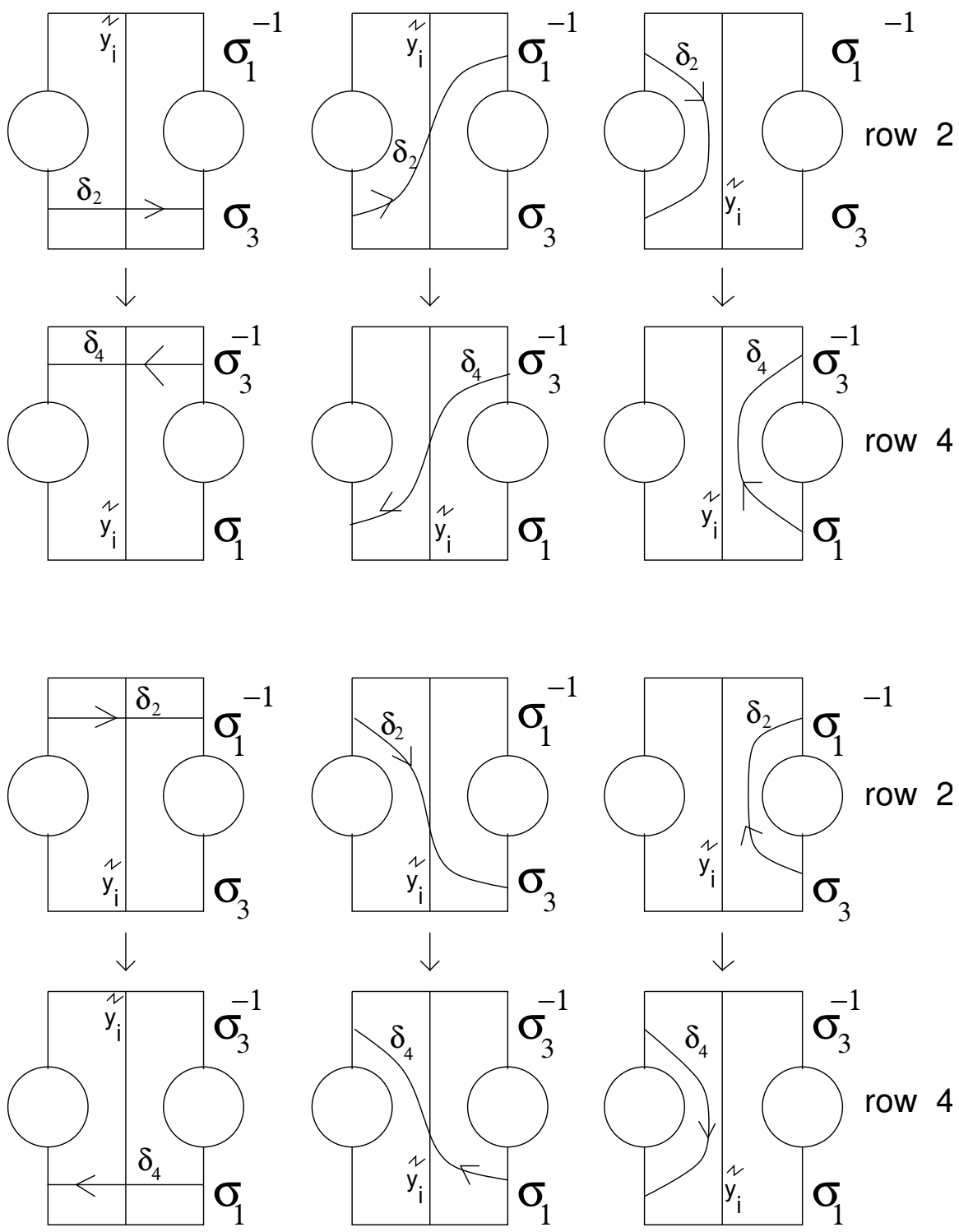

Figure 6. How to find cancelling loops in rows 2 and 4 .

These relations again determine a Coxeter group. It is well-known (see $[\mathbf{V}])$ that any such group surjects a finite group "without collapsing" - i.e., such that the orders of the images of the $\sigma_{i}$ 's and $\sigma_{i} \sigma_{j}$ 's are as given in (5)-(11). Then, arguing as in Case 1, we may find a non-peripheral fixed class in rows 2 and 5. 


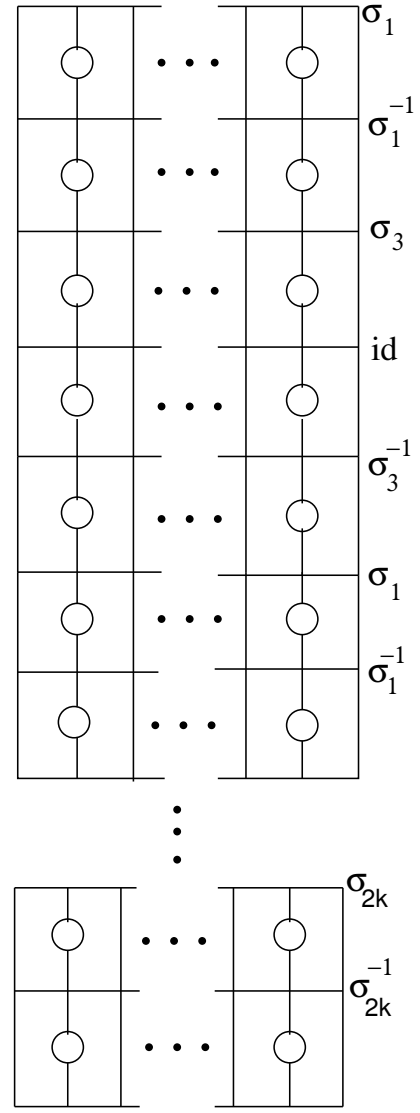

a

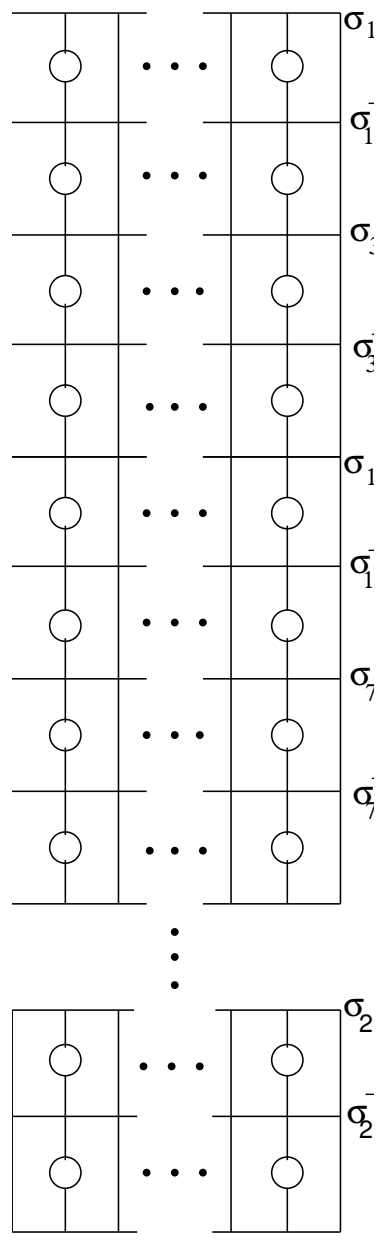

b

Figure 7. a. The cover for $n=2 k+1 \geq 9$. b. The cover for $n=2 k \geq 8$.

Case 3. $n=6$

Case 3a. $2 /|R \mu-\lambda|+1 /|\lambda|<1$.

Again, we need permutations $\sigma_{1}, \ldots, \sigma_{6}$ satisfying I-III. In this case we impose the additional conditions $\sigma_{2}=i d, \sigma_{3}=\sigma_{1}^{-1}, \sigma_{5}=i d$, and $\sigma_{6}=\sigma_{4}^{-1}$. 
Then conditions I-III reduce to:

$$
\begin{aligned}
\sigma_{1}^{R \mu-\lambda} & =1 \\
\left(\sigma_{1} \sigma_{4}\right)^{\lambda}=\left(\sigma_{4} \sigma_{1}\right)^{\lambda} & =1 \\
\sigma_{4}^{R \mu-\lambda} & =1 .
\end{aligned}
$$

These relations determine a triangle group, and we find a fixed class in rows 3 and 6 .

Case 3b. $|\lambda|>2$ and $|R \mu-3 \lambda| \geq|\lambda|$, or $\lambda$ is even (non-zero) and $|R \mu-3 \lambda| \geq$ 4.

When $n=3$, conditions I-III may be abelianized to obtain a cyclic group of order $|R \mu-3 \lambda|$. Specifically, they are satisfied by setting $\sigma_{1}=$ $(1,2, \ldots, R \mu-3 \lambda), \sigma_{2}=\sigma_{1}^{-2}$, and $\sigma_{3}=\sigma_{1}$. For $n=6$, we may "double" this cover: That is take $\sigma_{1}, \sigma_{2}, \sigma_{3}$ as above, and then set $\sigma_{4}=\sigma_{1}, \sigma_{5}=\sigma_{2}$, and $\sigma_{6}=\sigma_{3}$. Then we modify the corresponding cover $\tilde{M}(\mu, \lambda)$ of $M(\mu, \lambda)$ by making horizontal cuts in adjacent squares of row 3 and gluing the flaps back together as indicated by Fig. 8. If $\lambda$ is even, we make two non-adjacent cuts and glue the top of one to the bottom of the other. If $\lambda$ is odd, we make $(|\lambda|-1) / 2$ pairs of adjacent cuts and glue the top of the one cut to the bottom of the other cut in its pair. Now make the same cuts in row 6 , with the same identifications. Since rows 3 and 6 are fixed by $\tilde{D}_{x}, D_{x}$ still lifts to the modified $\tilde{M}_{h}(\mu, \lambda)$, and since the $\tilde{y}$ 's still project 6 to 1 onto $y, D_{y}$ lifts also; so $h$ lifts. Also, one may check that $\alpha^{\mu} \beta^{\lambda}$ still lifts, so $\tilde{M}_{h}(\mu, \lambda)$ remains a cover of $M_{h}(\mu, \lambda)$.

To see that $b_{1}\left(\tilde{M}_{h}(\mu, \lambda)\right)>0$, note that $\tilde{D}_{x}$ fixes rows 3 and 6 , so it is enough to find a non-peripheral loop in row 3 and add it to the corresponding loop in row 6 with opposite orientation. As in Case 1, the existence of such a non-peripheral loop follows from an Euler characteristic argument (or see Fig. 8).

Note that Case $3 \mathrm{a}$ or $3 \mathrm{~b}$ applies to all but finitely many $(\mu, \lambda)$ with $|\lambda|>1$. Case 4. $n=2 k \geq 8$.

Case 4a. $2 /|R \mu-2 \lambda|+1 /|\lambda|<1$. Set $\sigma_{2}=\sigma_{1}^{-1}, \sigma_{4}=\sigma_{3}^{-1}, \sigma_{5}=\sigma_{1}$, $\sigma_{6}=\sigma_{1}^{-1}$, and $\sigma_{2 i}=\sigma_{2 i-1}^{-1}$ for $i=4, \ldots, k$ (see Fig. 7b). Then, as in Case 2 , these relations determine a Coxeter group. We may find a non-peripheral fixed class in rows 2 and 4.

Case 4b. $|R \mu-\lambda| \leq 2$ We cannot guarantee, in this case, that there will always be a cover with $b_{1}>0$, but we shall show that there are at most finitely many exceptions.

We argue as in Case $3 \mathrm{~b}$. Take permutations $\sigma_{1}, \ldots, \sigma_{k}$, and consider the relations obtained by abelianizing conditions I-III. We claim that they can be satisfied by setting $\sigma_{1}=(1,2,3, \ldots, N)$, for some $\mathrm{N}$, and setting each 


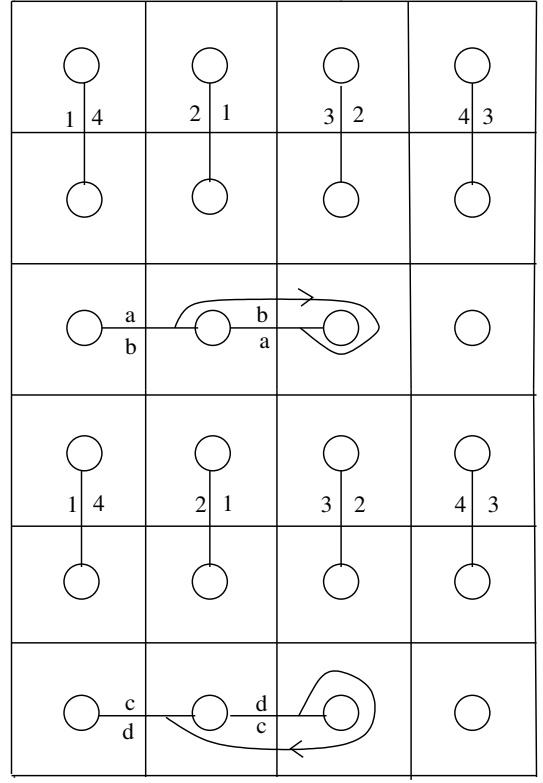

a

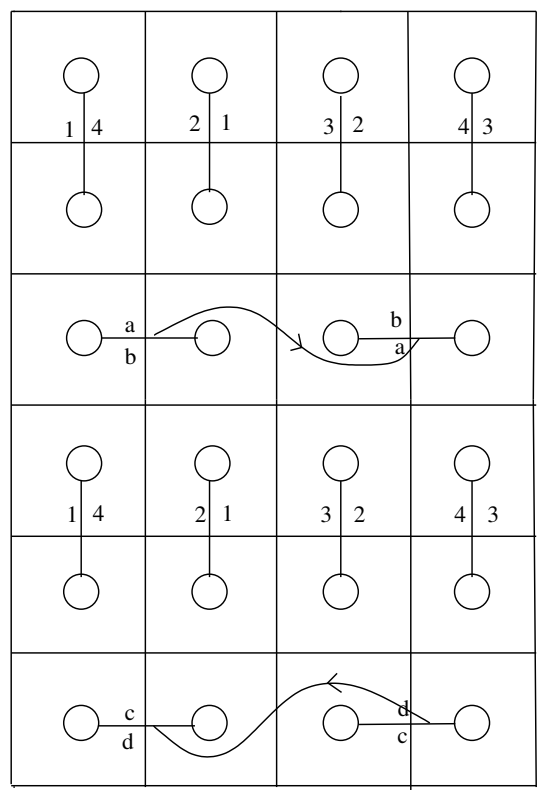

b

Figure 8. a. The cover and fixed class for $n=6, R \mu-3 \lambda=$ $4, \lambda=3$. b. The cover and fixed class for $n=6, R \mu-3 \lambda=$ $4, \lambda=2$.

$\sigma_{i}$ to an appropriate power of $\sigma_{1}$. We have already seen that this may be done when $k=3$.

The $\sigma_{i}$ 's must satisfy the following conditions:

$$
\begin{gathered}
\sigma_{1}^{R \mu-\lambda} \sigma_{2}^{\lambda}=1 \\
\sigma_{1}^{R \mu} \sigma_{2}^{R \mu-\lambda} \sigma_{3}^{\lambda}=1
\end{gathered}
$$

$$
\begin{gathered}
\sigma_{1}^{R \mu} \sigma_{2}^{R \mu} \cdots \sigma_{k-2}^{R \mu} \\
\sigma_{k-1}^{R \mu-\lambda} \sigma_{k}^{\lambda}=1 \\
\sigma_{1}^{R \mu+\lambda} \sigma_{2}^{R \mu} \cdots \sigma_{k-1}^{R \mu} \\
\sigma_{k}^{R \mu-\lambda}=1 \\
\sigma_{1} \sigma_{2} \cdots \sigma_{k}=1 .
\end{gathered}
$$


We shall assume that this system has a cyclic solution, so we may substitute $\sigma_{i}=\sigma_{1}^{e_{i}}$. Then, Equations (12)-(18) are equivalent to the following conditions on the exponents (all of the following equations in this case are taken $\bmod \mathrm{N})$ :

$$
\begin{gathered}
R \mu-\lambda+\lambda e_{2}=0 \\
R \mu+(R \mu-\lambda) e_{2}+\lambda e_{3}=0 \\
\vdots \\
R \mu+R \mu e_{2}+\cdots+R \mu e_{k-2}+(R \mu-\lambda) e_{k-1}+\lambda e_{k}=0 \\
R \mu+\lambda+R \mu e_{2}+\cdots+R \mu e_{k-1}+(R \mu-\lambda) e_{k}=0 \\
1+e_{2}+\cdots+e_{k}=0
\end{gathered}
$$

(22) and (23) imply that $\lambda=\lambda e_{k}$. Let us set $e_{k}=1$, eliminating Equation (22). Then, using (23), we may pair off (19) and (21) to deduce that $\lambda e_{2}=$ $\lambda e_{k-1}$, and we set $e_{2}=e_{k-1}$ to eliminate (21). Similarly, we set $e_{3}=e_{k-2}$, and so on. If $k$ is even, we are left with equations:

$$
\begin{gathered}
R \mu-\lambda+\lambda e_{2}=0 \\
R \mu+(R \mu-\lambda) e_{2}+\lambda e_{3}=0 \\
\vdots \\
R \mu+R \mu e_{2}+\cdots+(R \mu-\lambda) e_{k / 2-1}+\lambda e_{k / 2}=0 \\
R \mu+R \mu e_{2}+\cdots+(R \mu-\lambda) e_{k / 2}+\lambda e_{k / 2}=0 \\
2+2 e_{2}+\cdots+2 e_{k / 2}=0 .
\end{gathered}
$$

If we replace (28) by

$$
1+e_{2}+\cdots+e_{k / 2}=0
$$

then we may eliminate (27). Then solve for $\lambda e_{2}, \lambda^{2} e_{3}, \ldots, \lambda^{k / 2-1} e_{k / 2}$. By (29), we have:

(30) $\lambda^{k / 2-1}+\lambda^{k / 2-2}\left(\lambda e_{2}\right)+\lambda^{k / 2-3}\left(\lambda^{2} e_{3}\right)+\cdots+\lambda^{k / 2-1} e_{k / 2}=0$.

Substituting our solutions for $\lambda e_{2}, \lambda^{2} e_{3}$ and so on, we get the equation $N=0$ for some integer $N$; the system has a solution in $\mathbb{Z} / N \mathbb{Z}$.

If $k$ is odd, then our reduced system looks like:

$$
\begin{gathered}
R \mu-\lambda+\lambda e_{2}=0 \\
R \mu+(R \mu-\lambda) e_{2}+\lambda e_{3}=0
\end{gathered}
$$




$$
\begin{gathered}
R \mu+R \mu e_{2}+\cdots+(R \mu-\lambda) e_{(k-1) / 2}+\lambda e_{(k+1) / 2}=0 \\
R \mu+R \mu e_{2}+\cdots+(R \mu-\lambda) e_{(k+1) / 2}+\lambda e_{(k-1) / 2}=0 \\
2+2 e_{2}+\cdots+2 e_{(k-1) / 2}+e_{(k+1) / 2}=0 .
\end{gathered}
$$

Adding (33) and (34) gives a multiple of (35), so we may eliminate (34). Then we solve for $\lambda e_{2}, \lambda^{2} e_{3}, \ldots, \lambda^{(k-1) / 2} e_{(k+1) / 2}$. By (35), we have:

$$
\begin{array}{r}
2 \lambda^{(k-1) / 2}+2 \lambda^{(k-3) / 2}\left(\lambda e_{2}\right)+2 \lambda^{k-5 / 2}\left(\lambda^{2} e_{3}\right)+\cdots \\
+2 \lambda\left(\lambda^{(k-3) / 2} e_{(k-1) / 2}\right)+\lambda^{(k-1) / 2} e_{(k+1) / 2}=0 .
\end{array}
$$

And again we get a solution in $\mathbb{Z} / N \mathbb{Z}$ for some $N$.

Then, as in Case 3b, $M(\mu, \lambda)$ will have a cover with $b_{1}>0$, provided that $|N| \geq|\lambda|$ and $|\lambda|>2$. Solving for $N$, if $k$ is even, gives:

$$
N=\lambda^{k / 2-1}+\lambda^{k / 2-2}(\lambda-R \mu)+\lambda^{k / 2-3}\left[(\lambda-R \mu)^{2}-R \mu \lambda\right]
$$

$$
+\lambda^{k / 2-4}\left[(\lambda-R \mu)\left((\lambda-R \mu)^{2}-R \mu \lambda\right)-R \mu \lambda(\lambda-R \mu)-R \mu \lambda^{2}\right]+\cdots
$$

and if $k$ is odd:

$$
\begin{aligned}
& N=2 \lambda^{(k-1) / 2}+2 \lambda^{(k-3) / 2}(\lambda-R \mu)+2 \lambda^{(k-5) / 2}\left[(\lambda-R \mu)^{2}-R \mu \lambda\right] \\
& +2 \lambda^{(k-7) / 2}\left[(\lambda-R \mu)\left((\lambda-R \mu)^{2}-R \mu \lambda\right)-R \mu \lambda(\lambda-R \mu)-R \mu \lambda^{2}\right] \\
& +\cdots+1[. .] .
\end{aligned}
$$

We are supposing that $|R \mu-\lambda| \leq 2$. So for large $\mu$ or $\lambda, R \mu / \lambda \rightarrow 1$, and for $k$ even,

$$
\begin{array}{r}
N=o\left[\lambda^{k / 2-1}+\lambda^{k / 2-3}\left(-\lambda^{2}\right)+\lambda^{k / 2-4}\left(-\lambda^{3}\right)+\cdots\right] \\
=o\left[\lambda^{k / 2-1}-\lambda^{k / 2-1}-\lambda^{k / 2-1}-\cdots\right] .
\end{array}
$$

So if $k$ is even and $k \geq 8$, then for all but finitely many $\mu$ and $\lambda,|N|>|\lambda|$, and we are done. Similarly, if $k$ is odd and $k \geq 7$, then we are done. In the remaining cases, $|N|$ is given by:

$$
\begin{array}{r}
k=4,|N|=|R \mu-2 \lambda| \\
k=5,|N|=\left|(R \mu)^{2}-5 R \mu \lambda+5 \lambda^{2}\right| \\
k=6, \quad|N|=\left|(R \mu)^{2}-4 R \mu \lambda+3 \lambda^{2}\right| .
\end{array}
$$

One may check that each condition is satisfied by only finitely many relatively prime pairs $(\mu, \lambda)$ with $|R \mu-\lambda| \leq 2$. This concludes the proof in Case $4 \mathrm{~b}$.

Case 5. $n=7$, and $|\lambda|>1$.

Case 5a. $1 /|R \mu-\lambda|+1 /|\lambda|<2 / 3$ and $\left|(R \mu-2 \lambda)^{2}-2 \lambda^{2}\right|>2|R|$. 
We shall consider covers with $\sigma_{2}=i d, \sigma_{3}=\sigma_{1}^{-1}, \sigma_{6}=\sigma_{5}^{-1}$, and $\sigma_{7}=\sigma_{4}^{-1}$ (see Fig. 9a).

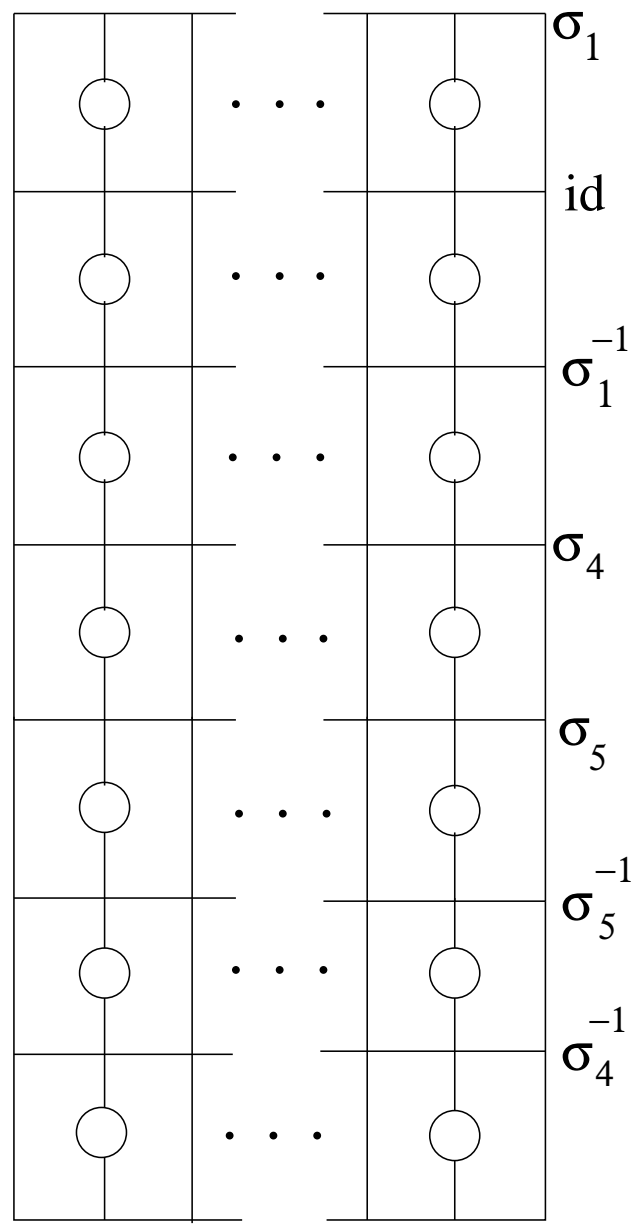

a

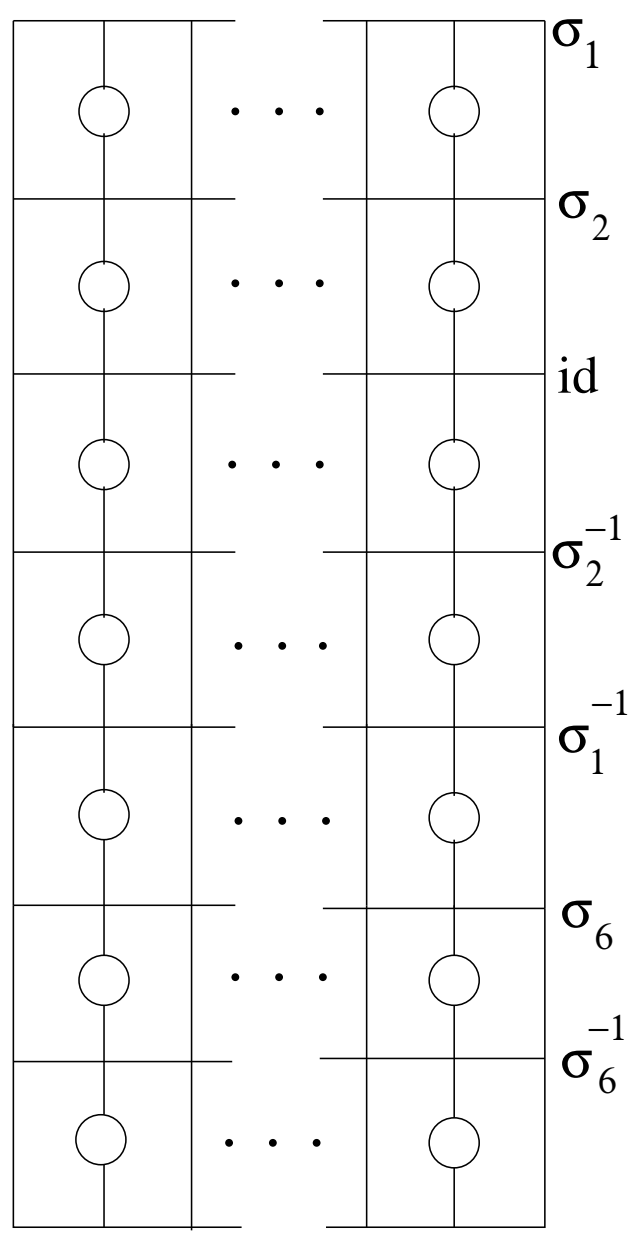

b

Figure 9. Two covers for $n=7$.

We obtain conditions:

(38)

$$
\begin{gathered}
{\left[\sigma_{4}, \sigma_{5}\right]=1} \\
\sigma_{1}^{R \mu-\lambda}=1 \\
\left(\sigma_{4} \sigma_{1}\right)^{\lambda}=1
\end{gathered}
$$




$$
\begin{gathered}
\sigma_{4}^{R \mu}\left(\sigma_{5} \sigma_{4}^{-1}\right)^{\lambda}=1 \\
\left(\sigma_{4} \sigma_{5}\right)^{R \mu} \sigma_{5}^{-2 \lambda}=1 \\
\left(\sigma_{1} \sigma_{4}\right)^{\lambda}=1 .
\end{gathered}
$$

Let us also assume for simplicity that $\sigma_{5}$ commutes with $\sigma_{1}$. Equations (38), (41) and (42) determine an abelian group $A$ of order $\left|(R \mu-2 \lambda)^{2}-2 \lambda^{2}\right|$; we must show that $\sigma_{4}^{2}$ is non-trivial in $A$. The elements $\sigma_{4}^{2}$ and $\sigma_{5}$ generate a subgroup $H$ of $A$ of index at most 2. If $\sigma_{4}^{2}=i d$, then $H$ is cyclic of order $\operatorname{gcd}(|\lambda|,|R \mu-2 \lambda|)$. Then $\left|(R \mu-2 \lambda)^{2}-2 \lambda^{2}\right|=|A| \leq 2|H|=2 \operatorname{gcd}(|\lambda|, \mid R \mu-$ $2 \lambda \mid)=2 \operatorname{gcd}(|\lambda|,|R|) \leq 2|R|$. So if

$$
\left|(R \mu-2 \lambda)^{2}-2 \lambda^{2}\right|>2|R|
$$

then $\sigma_{4}^{2} \neq i d$. Therefore, under our hypotheses, the relations generate a group which is isomorphic to the direct sum of a cyclic group with a hyperbolic triangle group. As in the previous cases, we may then find a nonperipheral fixed class (in rows 3 and 7 ), and we are done.

However, note that if $R=1$, then Equation (44) is false for all $(\mu, \lambda)$ satisfying

$$
(\mu+2 \lambda)^{2}-2 \lambda^{2}=1 .
$$

This is an example of Pell's equation, which has infinitely many solutions, and hence (44) may be false infinitely often.

Case 5b. $1 /|R \mu-2 \lambda|+1 /|\lambda|<2 / 3$ and $\left|(R \mu-\lambda)^{2}-2 \lambda^{2}\right|>2|R|$.

Let $\sigma_{3}=i d, \sigma_{4}=\sigma_{2}^{-1}, \sigma_{5}=\sigma_{1}^{-1}$, and $\sigma_{7}=\sigma_{6}^{-1}$ (see Fig. 9b). The conditions for a cover are:

$$
\begin{gathered}
{\left[\sigma_{1}, \sigma_{2}\right]=1} \\
\sigma_{1}^{R \mu}\left(\sigma_{2} \sigma_{1}^{-1}\right)^{\lambda}=1 \\
\left(\sigma_{1} \sigma_{2}\right)^{R \mu} \sigma_{2}^{-\lambda}=1 \\
\left(\sigma_{6} \sigma_{1}\right)^{\lambda}=1 \\
\sigma_{6}^{R \mu-2 \lambda}=1 \\
\left(\sigma_{1} \sigma_{6}\right)^{\lambda}=1 .
\end{gathered}
$$

For simplicity, suppose also that $\sigma_{2}$ commutes with $\sigma_{6}$. Then (45), (46), (47) determine an abelian group $B$ of order $\left|(R \mu-\lambda)^{2}-R \mu \lambda\right|$. If $\sigma_{1}^{2}=1$, then $|B| \leq 2 \operatorname{gcd}(|\lambda|,|R \mu-\lambda|)=2 \operatorname{gcd}(|\lambda|,|R|) \leq 2|R|$. Therefore, in this case, the group determined by conditions (45)-(50) is again the direct product 
of an abelian group with a hyperbolic triangle group, and we may find a non-peripheral fixed class in rows 5 and 7 .

Note that Case $5 \mathrm{a}$ or $5 \mathrm{~b}$ applies to all but finitely many surgeries where $|\lambda|>1$.

This concludes the proof of Theorem 1.3.

\section{Examples.}

We begin with the proof of Theorems 1.5 and 1.6 (see Section 1 for notation).

Lemma 3.1. Let $g=D_{y}^{5} D_{x}^{-1}$ and $h=D_{x} D_{y}$. Then $M_{-h}(\mu, \lambda) \cong M_{g}(\mu, \lambda-$ $\mu)$, and $M_{h^{2}}(\mu, \lambda) \cong M_{(-h)^{2}}(\mu, \lambda+\mu) \cong M_{g^{2}}(\mu, \lambda-\mu)$.

Proof. Recall that the mapping class group of the once-punctured torus is isomorphic to $S L_{2}(\mathbb{Z})$, under the identifications $D_{x} \rightarrow R=\left[\begin{array}{ll}1 & 1 \\ 0 & 1\end{array}\right]$ and $D_{y} \rightarrow L=\left[\begin{array}{ll}1 & 0 \\ 1 & 1\end{array}\right]$. Under these identifications, we compute that $h$ has monodromy matrix $\left[\begin{array}{ll}2 & 1 \\ 1 & 1\end{array}\right],(-1)$ has monodromy matrix $\left[\begin{array}{cc}-1 & 0 \\ 0 & -1\end{array}\right]$, and $g$ has monodromy matrix $\left[\begin{array}{ll}1 & -1 \\ 5 & -4\end{array}\right]$. The homeomorphisms $h^{2}$ and $(-h)^{2}$ have the same monodromy matrix, and hence are isotopic. Therefore $M_{h^{2}} \cong M_{(-h)^{2}}$. Also, $\left[\begin{array}{ll}-1 & 1 \\ -2 & 1\end{array}\right](-R L)\left[\begin{array}{ll}-1 & 1 \\ -2 & 1\end{array}\right]^{-1}=L^{5} R^{-1}$, so $g$ and $-h$ have conjugate monodromy matrices. It follows that $M_{-h} \cong M_{g}$, and $M_{(-h)^{2}} \cong M_{g^{2}}$.

It remains to determine the effect of these homeomorphisms on the framings. Computing the maps on $\pi_{1}(F)$ gives:

$$
(-h)_{\sharp}^{2}=\left(x^{-1} y x y^{-1}\right)\left(h_{\sharp}^{2}\right)\left(x^{-1} y x y^{-1}\right)^{-1} .
$$

Therefore the isotopy which takes $h^{2}$ to $(-h)^{2}$ twists $\partial F$ once in a counterclockwise manner, so the induced bundle homeomorphism sends $M_{h^{2}}(\mu, \lambda)$ to $M_{(-h)^{2}}(\mu, \lambda+\mu)$.

Let $f=D_{y}^{2} D_{x}^{-1}$. The bundle homeomorphism induced by conjugation preserves the framing, so $M_{-h}(\mu, \lambda) \cong M_{f(-h) f^{-1}}(\mu, \lambda)$. The homeomorphisms $f(-h) f^{-1}$ and $g$ have identical monodromy matrices, and hence are isotopic. We compute $g_{\sharp}=\left(y x^{-1} y^{-1} x\right) f(-h) f_{\sharp}^{-1}\left(y x^{-1} y^{-1} x\right)^{-1}$ so the isotopy from $f(-h) f^{-1}$ to $g$ twists $\partial F$ once in a clockwise manner. The induced bundle homeomorphism sends $M_{f(-h) f^{-1}}(\mu, \lambda)$ to $M_{g}(\mu, \lambda-\mu)$. So $M_{-h}(\mu, \lambda) \cong M_{g}(\mu, \lambda-\mu)$.

Likewise, $M_{f(-h)^{2} f^{-1}}(\mu, \lambda) \cong M_{g^{2}}(\mu, \lambda-2 \mu)$. Thus

$$
M_{h^{2}}(\mu, \lambda) \cong M_{(-h)^{2}}(\mu, \lambda+\mu) \cong M_{f(-h)^{2} f^{-1}}(\mu, \lambda+\mu) \cong M_{g^{2}}(\mu, \lambda-\mu) .
$$

Proof of Theorem 1.5. This is an immediate consequence of Lemma 3.1 and Theorem 1.3. 
Proof of Theorem 1.6. We have $M(2 \mu, \lambda) \cong M_{h}(2 \mu, \lambda)$, which is double covered by $M_{h^{2}}(\mu, \lambda) \cong M_{g^{2}}(\mu, \lambda-\mu)$. So it is enough to show that $M_{g^{2}}(\mu, \lambda-\mu)$ is virtually $\mathbb{Z}$-representable. By Theorem 1.3, we are done unless

$$
1 /|-2 \mu-(\lambda-\mu)|+1 /|-2 \mu-2(\lambda-\mu)|+1 /|\lambda-\mu| \geq 1
$$

or, simplifying:

$$
1 /|\mu+\lambda|+1 /|2 \lambda|+1 /|\mu-\lambda| \geq 1 .
$$

By [B3], $M(2 \mu, \lambda)$ is virtually $\mathbb{Z}$-representable if $2 \mu$ is divisible by 4 ; hence we may assume $\mu$ is odd. Also, since $\operatorname{gcd}(2 \mu, \lambda)=1$, we may assume $\lambda$ is odd, and, assuming $(\mu, \lambda) \neq( \pm 1,1),|\lambda| \neq|\mu|$. It follows that

$$
\begin{aligned}
& |\mu-\lambda| \geq 2 \\
& |\mu+\lambda| \geq 2 .
\end{aligned}
$$

The only simultaneous solutions to inequalities (51), (52) and (53) with $\mu$ and $\lambda$ odd are: $(\mu, \lambda)= \pm(-3,1)$ and $\pm(3,1)$. So the only possible exceptions to Theorem 1.6 are $M(-6,1) \cong M(6,1)$ and $M(2,1) \cong M(-2,1)$. The virtual $\mathbb{Z}$-representability of these manifolds may be verified with either of the computer programs GAP or Snappea.

We now turn to the proof of Theorem 1.4.

Proof of Theorem 1.4. Let $g$ and $h$ be as in the statement of Lemma 3.1, let $f=h^{18}$, and let $i=D_{x}^{2} D_{y}^{-4} D_{x} D_{y}^{-4} D_{x}$. Both $h^{3}$ and $i$ have monodromy matrix $\left[\begin{array}{cc}13 & 8 \\ 8 & 5\end{array}\right]$; hence $h^{3}$ and $i$ are isotopic. By arguments similar to those used in the proof of Lemma 3.1, we compute that $M_{h^{3}}(\mu, \lambda) \cong M_{i}(\mu, \mu+\lambda)$. Therefore $M_{f}(\mu, \lambda) \cong M_{i^{6}}(\mu, \lambda+6 \mu)$. Hence by Theorem 1.3 iii, $M_{f}(\mu, \lambda)$ is virtually $\mathbb{Z}$-representable if

$$
1 /|6 \mu-\lambda|+1 /|6 \mu+\lambda|<1 .
$$

By Lemma 3.1 we have $M_{f}(\mu, \lambda) \cong M_{g^{18}}(\mu, \lambda-9 \mu)$. Hence by Theorem $1.3 \mathrm{ii}, M_{f}(\mu, \lambda)$ is virtually $\mathbb{Z}$-representable if

$$
1 /|9 \mu+\lambda|+1 /|2 \lambda|+1 /|9 \mu-\lambda|<1 .
$$

The only simultaneous solutions to the inequalities 54 and 55 have $\mu=$ 0 . The proof is completed by noting that $M(0,1)$ has positive first Betti number, as it is a torus bundle over $S^{1}$.

We remark that the same methods may be applied to many other examples of once-punctured torus bundles, to show that all but finitely many surgeries are virtually $\mathbb{Z}$-representable. The idea is to start with a monodromy $f$ to which Theorem 1.3 i or ii applies. Since $L^{4}$ and $R$ generate a finite-index subgroup of $S L_{2}(\mathbb{Z})$, there exists an integer $\ell$ such that $f^{\ell}$ is 
isotopic to a $g$ satisfying the hypotheses of Theorem 1.3 iii. Usually Theorem 1.3 will then imply that all but finitely many surgeries on $M_{f \ell}$ are virtually $\mathbb{Z}$-representable.

\section{References}

[B1] M. Baker, Covers of Dehn fillings on once-punctured torus bundles, Proc. Amer. Math. Soc., 105 (1989), 747-754.

[B2] Covers of Dehn fillings on once-punctured torus bundles II, Proc. Amer. Math. Soc., 110 (1990), 1099-1108.

[B3] - On coverings of figure eight-knot surgeries, Pacific J. Math., 150 (1991), 215-228.

[Bart] A. Bart, Surface groups in surgered manifolds, to appear in Topology.

[BZ] S. Boyer and X. Zhang, Virtual Haken 3-manifolds and Dehn filling, Topology, 39 (2000), 103-114.

[CJR] M. Culler, W. Jaco and H. Rubinstein, Incompressible surfaces in once-punctured torus bundles, Proc. London Math. Soc., 45(3) (1982), 385-419.

[FH] W. Floyd and A. Hatcher, Incompressible surfaces in punctured torus bundles, Topology and it Applications, 13 (1982), 263-282.

[H] J. Hempel, Coverings of Dehn fillings of surface bundles, Topology and its Applications, 24 (1986), 157-170.

[KL] S. Kojima and D. Long, Virtual Betti numbers of some hyperbolic 3-manifolds, A Fete of Topology, Academic Press, 1988.

[M] S. Morita, Finite coverings of punctured torus bundles and the first Betti number, Sci. Papers College Arts Sci., Univ Tokyo, 35 (1986), 109-121.

[N] A. Nicas, An infinite family of hyperbolic non-Haken 3-manifolds with vanishing Whitehead groups, Math. Proc. Camb. Phil. Soc., 99 (1986), 239-246.

[V] E.B. Vinberg, Groups defined by periodic paired relations, Sbornik: Mathematics, 188 (1997), 1269-1278.

Received June 15, 1998.

University of Texas AT Austin

Austin, Texas 78712

RICE UNIVERSITY

Houston, Texas 77005-1892

E-mail address: mastersj@rice.edu 